\title{
Doctors' knowledge of the appropriate use and route of administration of antidotes in the management of recreational drug toxicity
}

\author{
S Lidder, ${ }^{1}$ H Ovaska, ${ }^{1}$ J R H Archer, ${ }^{2}$ S L Greene, ${ }^{1}$ A L Jones, ${ }^{3}$ P I Dargan, ${ }^{1}$ D M Wood ${ }^{1}$
}

${ }^{1}$ Guy's and St Thomas Poisons Unit, Guy's and St Thomas' NHS Foundation Trust, London, UK;

${ }^{2}$ St George's, University of London, London, UK; ${ }^{3}$ University of Newcastle, Newcastle, NSW, Australia

Correspondence to: Dr D Wood, Guy's and St Thomas Poisons Unit, Avonley Road, London SE14 5ER, UK; David.Wood@gstt.nhs.uk

Accepted 10 May 2008

\section{ABSTRACT}

Background: Specific antidotes (eg, naloxone, flumazenil, cyproheptadine and benzodiazepines) are available for the management of certain recreational drug-induced toxicities. Some controversies surround the use of some of these antidotes, especially flumazenil in benzodiazepine toxicity. There are no previously published data on doctors' knowledge of the use of these specific antidotes. Methods: A questionnaire survey was designed to determine internal/emergency medicine doctors' knowledge of the appropriate use of antidotes in the management of clinical scenarios of acutely poisoned patients. For nine simulated clinical scenarios of acute toxicity from recreational drugs (benzodiazepines, cocaine, N-methyl---(3, 4-methylene-dioxyphenyl)-2-aminopropane (MDMA)-induced serotonin toxicity and opioids), they were asked to indicate whether the suggested antidote and route of administration were correct.

Results: 42 physicians of all grades completed the questionnaire. The mean correct score was 5.4 (SD 1.1) (median 6, interquartile range 5-7). The percentages correct for the various clinical scenarios were $68.3 \%$ for opioid toxicity, $81 \%$ for benzodiazepine toxicity, $28.6 \%$ for MDMA-induced serotonin toxicity and $70.2 \%$ for cocaine toxicity. Doctors were more likely to record an answer of "unsure" for the use of cyproheptadine in ST serotonin toxicity $(28.6 \%)$ compared with the use of the other antidotes $(1.4 \% ; p<0.001)$.

Conclusion: Knowledge of the appropriate use of antidotes in recreational drug toxicity is not consistent, with poorer knowledge on the use of newer antidotes such as cyproheptadine in serotonin toxicity. Education is required both to increase overall knowledge on the use of specific antidotes in the management of recreational drug-induced toxicity, as well as focusing on newer antidotes such as cyproheptadine.

The use of recreational drugs in the UK is common, and overall there has been an increase in their use, with the highest use in those aged under 45 years. ${ }^{1}$ Routine toxicological screening is typically not undertaken in patients presenting to the emergency department with toxicity following the use of recreational drugs, because there is limited or no availability of "real-time results" to assist clinicians in their management decisions. However, recreational drugs typically tend to cause symptoms and/ or signs that can help a clinician in determining what drug or drugs have been ingested.

The mainstay of the treatment of patients with toxicity following the use of recreational drugs is meticulous supportive care and management of complications if they occur. In addition, there are some specific antidotes and/or treatments that may be useful in the management of recreational drug toxicity. These include naloxone for opioid toxicity, flumazenil for benzodiazepine toxicity, cyproheptadine for serotonin toxicity and benzodiazepines for cocaine toxicity. ${ }^{2-5}$ However, there is controversy concerning the widespread use of some of these antidotes, especially flumazenil in benzodiazepine toxicity. ${ }^{36}$ In addition, some antidotes may be administered by more than one route, for example intramuscular or intravenous naloxone. Not only does each antidote need to be administered correctly, but they should also be administered by the correct route, in the correct dose and in the right clinical circumstances, to minimise the risk of unwanted adverse effects.

No studies have been published on doctors' knowledge of the appropriate use of antidotes and their routes of administration in the management of patients with recreational drug toxicity. We therefore designed a questionnaire survey to determine adult internal medicine and emergency physicians' knowledge of the appropriate use and route of administration of various antidotes in the management of recreational drug toxicity.

\section{METHODS \\ Study participants}

Medical staff of all grades in general (internal) medicine and emergency medicine were recruited before attending one of three clinical toxicology training sessions.

\section{Questionnaire survey}

Participants were asked to indicate if the antidote/ treatment and route of administration specified for nine clinical scenarios of acute recreational drug toxicity were correct or not (table 1). The appropriateness or not of both the antidote/ treatment and the route of administration for each of these clinical scenarios was judged by a panel of clinical toxicologists, which comprised the senior authors (DW/PD/SG/AJ). The answers of each participant were compared with those of the panel of clinical toxicologists and a score of one mark per scenario was given when there was concordance in the answers.

\section{Outcome measures}

The primary outcome measure was identification that an appropriate antidote and route of administration had been given for the individual clinical scenario. 
Table 1 Clinical scenarios of acutely poisoned patients with suggested antidote for the individual presentation and the potential route of administration

\begin{tabular}{|c|c|}
\hline Clinical scenario & Antidote/treatment \\
\hline $\begin{array}{l}\text { 18-Year-old man with ingestion of } 10 \text { ecstasy tablets. Temperature } 39.6^{\circ} \mathrm{C} \text {, HR } 150 \text {, spontaneous } \\
\text { clonus and hyperreflexia }\end{array}$ & PO cyproheptadine \\
\hline $\begin{array}{l}\text { 52-Year-old woman with epilepsy presents after ingestion of temazepam with a GCS of } 8 \text { and } \\
\text { normal BP }\end{array}$ & IV flumazenil \\
\hline 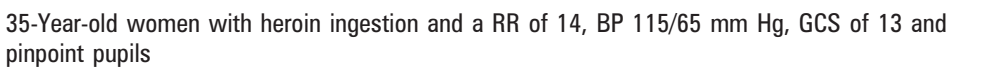 & IM naloxone \\
\hline $\begin{array}{l}\text { 42-Year-old man with ingestion of two lines of cocaine presenting with ischaemic chest pain, ECG } \\
\text { changes (ST depression) and a BP of } 175 / 95 \mathrm{~mm} \mathrm{Hg}\end{array}$ & IV metoprolol \\
\hline $\begin{array}{l}\text { 24-Year-old man ingested an unknown quantity of codeine and has RR of } 6 \text {, BP of } 105 / 70 \mathrm{~mm} \mathrm{Hg} \\
\text { and GCS of } 4\end{array}$ & IV naloxone \\
\hline $\begin{array}{l}\text { 29-Year-old with ingestion of amphetamine and temperature of } 38.5^{\circ} \mathrm{C} \text {, normal tone and reflexes } \\
\text { and no clonus }\end{array}$ & PO cyproheptadine \\
\hline $\begin{array}{l}\text { 37-Year-old woman presents after ingestion of temazepam and other unknown tablets with a GCS } \\
\text { of } 8, \mathrm{HR} 120, \mathrm{BP} 85 / 45 \mathrm{~mm} \mathrm{Hg}\end{array}$ & IV flumazenil \\
\hline $\begin{array}{l}\text { 24-Year-old man ingested an unknown quantity of codeine and has RR of } 6 \text {, BP of } 105 / 70 \mathrm{~mm} \mathrm{Hg} \\
\text { and GCS of } 4 \text {. He has IV fluids in-situ }\end{array}$ & IM naloxone \\
\hline $\begin{array}{l}\text { 37-Year-old man with ingestion of one line of cocaine presenting with agitation and BP } \\
180 / 85 \mathrm{~mm} \mathrm{Hg} \text { and heart rate of } 140\end{array}$ & IV diazepam \\
\hline
\end{tabular}

BP, blood pressure; ECG, electrocardiogram; GCS, Glasgow coma scale score; HR, heart rate; IM, intramuscular; IV, intravenous; $\mathrm{PO}$, by mouth; RR, respiration rate.

\section{Primary data analysis}

Data are presented as mean (SD), along with median and interquartile range, when appropriate. Comparison of data was undertaken by unpaired Student's t test. Statistical analysis was undertaken by SPSS, version 10.

\section{RESULTS}

A total of 42 doctors of all grades in general and emergency medicine attending three clinical toxicology training sessions were recruited and completed survey questionnaires were returned by all participants. The mean (SD) score for all doctors completing the case scenarios on the use of antidotes/treatment in recreational drug toxicity was 5.4 (1.1) and the median (interquartile range) was $6(5-7)$. The percentage of correct answers for each individual scenario is shown in fig 1 .

The individual scenarios could be "grouped" as opioid toxicity (scenarios 3, 5 and 8), benzodiazepine toxicity (scenarios 2 and 7), N-methyl-L-(3, 4-methylene-dioxyphenyl)-2-aminopropane (MDMA)-induced serotonin toxicity (scenarios 1 and 6) and cocaine toxicity (scenarios 4 and 9). The percentage of answers that were correct for the various groups of clinical scenarios were $68.3 \%, 81 \%, 28.6 \%$ and $70.2 \%$ for opioid toxicity, benzodiazepine toxicity, MDMA-induced serotonin toxicity and cocaine toxicity, respectively (fig 2). Participants were more likely to record an answer of "unsure or don't know" for the use of cyproheptadine in MDMA-induced serotonin toxicity $(28.6 \%)$ compared with the use of the antidote in the other

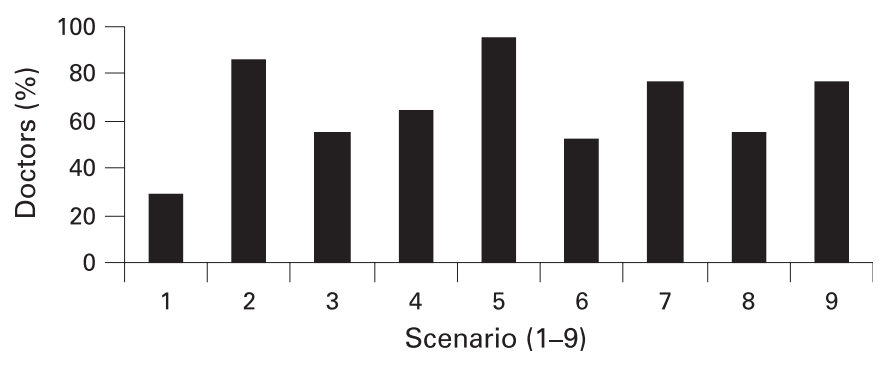

Figure 1 Percentage of doctors correctly identifying that the antidote and route of administration for a scenario of recreational drugs of abuse toxicity was correct or not. scenarios (opioid toxicity, cocaine toxicity and benzodiazepine toxicity) $(1.4 \%$; $<0.001)$.

\section{DISCUSSION}

The aim of the study was to determine physicians' knowledge on the appropriate use and routes of administration of the various antidotes/treatments that may be used in the management of recreational drug toxicity. We used nine clinical scenarios of acute recreational drug toxicity, which are commonly seen in the emergency department to test physicians' knowledge of the appropriate use of antidotes/management in each scenario. We have demonstrated that overall doctors had some knowledge on the use and route of the various antidotes, although their knowledge differed between antidotes, with less knowledge of newer antidotes such as cyproheptadine.

There is published literature about the efficacy of the specific antidotes used in the clinical scenarios in this study in the management of patients with recreational drug toxicity. However, there are some controversies about the use of these antidotes and in particular the potential for adverse effects, including mortality following their use. In the discussion we would like to raise some of these issues and also look at the recommendations for their use.

Naloxone is a specific opioid antagonist that can be used to reverse the symptoms of opioid toxicity, such as coma and respiratory depression, and its use in these situations is recommended by the National Institute for Health and Clinical Excellence (NICE). ${ }^{7}$ There is no "standard" dose of naloxone that should be administered, although the British National Formulary recommends bolus doses of $0.8-2 \mathrm{mg}$, repeated as required. ${ }^{8}$ Although it is a relatively safe antidote, there have been reports of adverse effects following use, including acute withdrawal symptoms in opioid-dependent users, seizures, arrhythmias and acute pulmonary oedema. ${ }^{2}$ It is possible that the acute pulmonary oedema that has been reported is due either to the opioid toxicity itself, underlying cardiorespiratory disease or that the reversal of opioid toxicity with naloxone improves respiratory effort and therefore unmasks opioid-induced pulmonary oedema. Similarly, seizures and cardiac arrhythmias following naloxone use may be due to the opioid toxicity directly, co-ingested substances such as 


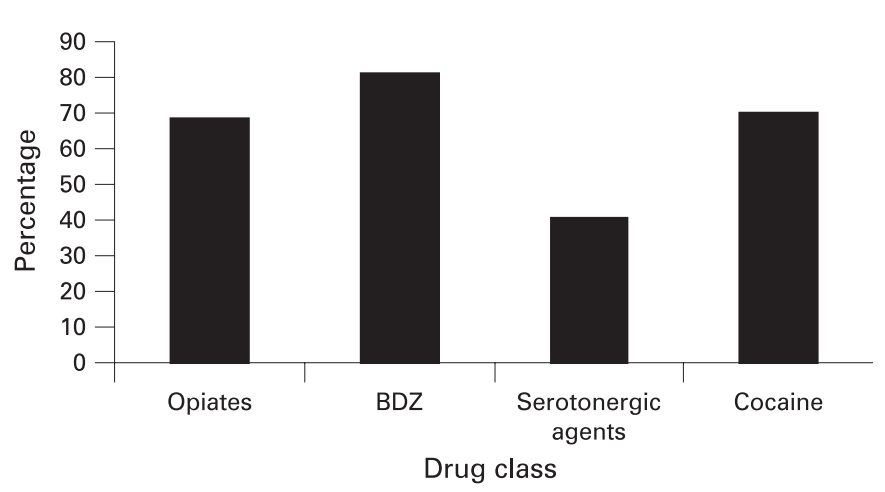

Figure 2 Percentage of answers correct for each type of scenario of recreational drugs of abuse toxicity (opioid toxicity, benzodiazepine toxicity, N-methyl-L-(3, 4-methylene-dioxyphenyl)-2-aminopropaneinduced serotonin toxicity, cocaine toxicity). BDZ, benzodiazepine.

cocaine or underlying cardiac disease. Precipitating acute withdrawal in opioid-dependent users cannot only be difficult to manage within the emergency department, with patients becoming aggressive and violent, but is also distressing and unpleasant for patients. The frequency at which acute withdrawal is precipitated is not clear, because the outcome measures to determine the development of acute withdrawal vary between studies, but it has been reported to vary between $7 \%$ and $47 \%$. The administration of large bolus doses is more likely to precipitate acute withdrawal symptoms, and therefore small titrated intravenous bolus doses are recommended for the management of patients with opioid toxicity as they are less likely to cause acute withdrawal. ${ }^{7}$ The optimum route of administration of naloxone is controversial; two studies comparing intravenous and intramuscular/subcutaneous administration have failed to answer this question adequately and complication rates were not well described in the studies. ${ }^{9}{ }^{10}$ However, in view of the potential for unpredictable and erratic absorption of naloxone following intramuscular administration and therefore the unpredictable reversal of opioid toxicity and the potential for more significant acute opioid withdrawal, the intravenous route remains the route of choice unless intravenous access is impossible. ${ }^{2}$ Given the potential for adverse effects following inappropriate route of administration and/or dosing regimens, flowcharts for the use of naloxone in the management of opioid toxicity have been developed to try and minimise the development of these adverse effects. ${ }^{2}$

Although flumazenil is licensed in the UK for the management of iatrogenic benzodiazepine overdose, for example in reversing sedation for upper gastrointestinal endoscopy, it is not licensed for use in the management of deliberate self-poisoningrelated benzodiazepine toxicity. ${ }^{7}$ Although clinicians may currently use it outside of its current product licence in the UK, previous guidance issued by NICE recommends that "only clinicians who have been explicitly trained in the use of flumazenil for the treatment of benzodiazepine poisoning should undertake to administer flumazenil". ' In addition, some studies have suggested that as benzodiazepine overdoses tend to be well tolerated by patients, specific antidotal treatment is not required. ${ }^{6}$ Similar to naloxone use in opioid-dependent users, flumazenil use has been demonstrated to precipitate an acute benzodiazepine withdrawal syndrome in chronic users. ${ }^{11}$ Of more concern is that there have been previous reports of seizures and potentially fatal arrhythmias. ${ }^{12}$ These potential serious adverse effects were acknowledged in the NICE guidance, and are more likely to occur if the patient has underlying epilepsy or has co-ingested substance(s) known to cause seizures and/or cardiac arrhythmias in overdose, for example tricyclic antidepressants. ${ }^{11} 1314$ There is also an increased incidence of vomiting and agitation. Although a more recent review has suggested that flumazenil is beneficial in the management of benzodiazepine overdose, the authors acknowledged that there was a "lack of uniformity in the reporting of coma scales" and differences in the presentation of the data in the studies included. ${ }^{3}$ In addition, the clinical significance of flumazenil use is not clear, because few of the studies have reported outcomes such as a reduction in the need for mechanical ventilation or endotracheal intubation rates.

Serotonin toxicity is a recognised unwanted effect following the use of serotoninergic agents such as MDMA. The diagnostic criteria and features of serotonin toxicity have recently been well described, aiding clinicians in its diagnosis and management. ${ }^{4}$ Controversies about the management of serotonin toxicity persist, especially about the potential beneficial effects of agents such as dantrolene. The initial treatment of serotonin toxicity is cessation of the precipitating drug, if appropriate, and meticulous supportive care and management of the complications as they occur. For patients with mild-to-moderate serotonin toxicity, additional treatment with cold intravenous fluids and intravenous benzodiazepines may be required for the management of hyperthermia and agitation. ${ }^{15}$ Those patients with more severe features of serotonin toxicity, in particular hyperpyrexia, require more aggressive treatment to prevent significant morbidity and mortality. Previously, dantrolene was suggested for the management of serotonin toxicity, as a result of its actions on the sarcoplasmic reticulum of muscle, leading to reduced muscle activity and therefore a reduction in temperature. Animal studies, however, have demonstrated that dantrolene has no benefit on outcome in serotonin toxicity and there have been reports of fatalities following its use in serotonin toxicity. ${ }^{16}{ }^{17}$ Cyproheptadine is a $5 \mathrm{HT}_{2 \mathrm{~A}}$ antagonist, which has been shown to be beneficial in the management of serotonin toxicity, because it specifically targets the excess serotonin seen both centrally and peripherally in serotonin toxicity. ${ }^{45}$ It is currently only available as an oral preparation and although unlicensed in the UK for the management of serotonin toxicity, we would recommend an initial dose of $12 \mathrm{mg}$, followed by $2 \mathrm{mg}$ every $2 \mathrm{~h}$ until symptoms settle to a maximum of $32 \mathrm{mg}$ in $24 \mathrm{~h}$.

Unlike benzodiazepine, opioid and serotonin toxicity, there is no specific "antidote" for the management of cocaine toxicity. Typical symptoms of cocaine toxicity are sympathomimetic in nature and include tachycardia and arrhythmias, hypertension, hyperthermia and anxiety and agitation. The vasoconstrictive actions of cocaine are also associated with ischaemia, and in particular acute myocardial ischaemia and infarction; mesenteric ischaemia and ischaemic cerebrovascular events are well recognised..$^{12}$ As there is no specific antidote for cocaine toxicity, treatment is meticulous supportive care and the management of specific complications as they occur. This includes the use of benzodiazepines for significant agitation and anxiety, although larger doses than normal may be required. ${ }^{12}$ The risk of myocardial ischaemia and infarction following cocaine use is greatest within the first hour after use and is predominately due to coronary artery vasoconstriction. In atherosclerotic-induced myocardial ischaemia, the recognised treatment regimen consists of oxygen, aspirin, morphine, nitrates and beta-blockers. However, in cocaine-induced myocardial ischaemia, the use of beta-blockers in particular has been demonstrated to be associated with an increased risk of fatalities, worsening of 
coronary artery vasoconstriction and potentially paradoxical worsening of any associated hypertension. ${ }^{18-20}$ Therefore, the use of beta-blockers in this setting is absolutely contraindicated. In addition to the use of oxygen, morphine and aspirin, both benzodiazepine and nitrate use should be considered in terms of reversing coronary artery vasoconstriction. In particular, studies have shown that benzodiazepines are as effective as nitrates in reversing cocaine-induced coronary artery vasoconstriction. ${ }^{5}$

Teaching on recreational drugs often does not form part of the undergraduate training for doctors in the UK. There has been considerable concern from clinical pharmacologists following the publication of Tomorrow's doctors by the General Medical Council in $1993 .{ }^{21}$ Following the publication of this document, dedicated clinical pharmacology and therapeutics, including clinical toxicology, teaching courses were removed from the majority of undergraduate medical curricula. In addition, few doctors undertake postgraduate training in clinical toxicology. This may therefore reflect in the limited knowledge of doctors in terms of newer antidotes, such as cyproheptadine for the management of serotonin toxicity. Previous studies have demonstrated that short courses in clinical toxicology, lasting only a day or two, can produce a sustained improvement in doctors' knowledge of issues relating to clinical toxicology. ${ }^{22}$

Appropriate knowledge and understanding of the correct use of antidotes and their route(s) of administration by physicians treating patients with recreational drug toxicity is essential to maximise potential benefit, while minimising the potential for adverse or unwanted effects. Therefore it is important that more directed education concerning the management of recreational drug toxicity is provided to both undergraduate and postgraduate medical trainees. Although this information can most efficiently be provided by clinical toxicologists, it is important that this information is provided to all clinicians involved in the management of these patients and updated regularly to include novel antidotes and/or routes of administration as they become available.

\section{Competing interests: None.}

Contributors: DMW had the initial concept for the study and designed the study questionnaire; PID, ALJ, HO, JRHA and SLG revised and finalised the design of the study questionnaire and the study protocol. DMW and PID undertook the study and DMW undertook the initial data analysis. SL prepared the first draft of the manuscript, initial review of this was undertaken by DMW and all authors contributed to the revised manuscript.

\section{REFERENCES}

1. United Nations Office on Drugs and Crime. 2007 World Drug Report. United Nations publication E. 07.XI.5 (ISBN 978-92-1-148222-5). www.unodc.org/ (accessed September 2008).

2. Clarke S, Dargan PI, Jones AL. Naloxone in opioid poisoning: walking the tightrope. Emerg Med J 2005;22:612-16.

3. Ngo AS, Anthony CR, Samuel M, et al. Should a benzodiazepine antagonist be used in unconscious patients presenting to the emergency department? Resuscitation 2007; 74:27-37.

4. Boyer EW, Shannon M. The serotonin syndrome. N Engl J Med 2005;352:1112-20.

5. Baumann BM, Perrone J, Hornig SE, et al. Randomized, double-blind, placebocontrolled trial of diazepam, nitroglycerin, or both for treatment of patients with potential cocaine-associated acute coronary syndromes. Acad Emerg Med 2000; 7:878-85

6. Carvalho C, Walker DA. Coma cocktail: a role for flumazenil? Br J Hosp Med (Lond) 2007:68:112.

7. National Institute for Health and Clinical Excellence. Self-harm. The short-term physical and psychological management and secondary prevention of self-harm in primary and secondary care. 2004. www.nice.org.uk/CG016NICEguideline laccessed September 2008).

8. British National Formulary. Flumazenil. http://www.bnf.org.uk/bnf/bnf/56/129247. htm (accessed 29 September 2008).

9. Sporer KA, Firestone J, Isaacs M. Out of hospital treatment of opioid overdoses in an urban setting. Acad Emerg Med 1999;3:660-7.

10. Wanger K, Brough L, MacMillan I, et al. Intravenous versus subcutaneous naloxone for out of hospital management of presumed opioid toxicity. Acad Emerg Med 1998:5:293-9.

11. Weinbroum AA, Flaishon R, Sorkine P, et al. A risk-benefit assessment of flumazenil in the management of benzodiazepine overdose. Drug Saf 1997;17:181-96.

12. Greene SL, Dargan PI, Jones AL. Acute poisoning: understanding $90 \%$ of cases in a nutshell. Postgrad Med J 2005;81:204-16.

13. The Flumazenil in Benzodiazepine Intoxication Multicenter Study Group. Treatment of benzodiazepine overdose with flumazenil. Clin Ther 1992;14:978-95.

14. Lheureux $\mathbf{P}$, Vranckx $\mathrm{M}$, Leduc $\mathrm{D}$, et al. Flumazenil in mixed benzodiazepine/tricyclic antidepressant overdose: a placebo-controlled study in the dog. Am J Emerg Med 1992;10:184-8.

15. Gillman PK. The serotonin syndrome and its treatment. J Psychopharmacol 1999:13:100-9.

16. Nisijima K, Yoshino T, Yui K, et al. Potent serotonin (5-HT)(2A) receptor antagonists completely prevent the development of hyperthermia in an animal model of the 5-HT syndrome. Brain Res 2001;890:23-31.

17. Kline SS, Mauro LS, Scala-Barnett DM, Zick D. Serotonin syndrome versus neuroleptic malignant syndrome as a cause of death. Clin Pharm 1989;8:510-14.

18. Albertson TE, Dawson A, de Latorre F, et al. American Heart Association; International Liaison Committee on Resuscitation. TOX-ACLS: toxicologic-oriented advanced cardiac life support. Ann Emerg Med 2001;37(4 Suppl):S78-90.

19. Lange RA, Cigarroa RG, Flores ED, et al. Potentiation of cocaine-induced coronary vasoconstriction by beta-adrenergic blockade. Ann Intern Med 1990;112:897-903.

20. Sand IC, Brody SL, Wrenn KD, et al. Experience with esmolol for the treatment of cocaine-associated cardiovascular complications. Am J Emerg Med 1991;9:161-3.

21. Maxwell S, Walley T, BPS Clinical Section Committee. Teaching safe and effective prescribing in UK medical schools: a core curriculum for tomorrow's doctors. Br J Clin Pharmacol 2003;55:496-503

22. Wood DM, Greene SL, Jones AL, et al. Gut decontamination of acutely poisoned patients—what do doctors really know about it? Emerg Med J 2007;24:774-5. 